\title{
Optical Spectroscopy Integrated with Environmental Scanning Transmission Electron Microscope: A Comprehensive In Situ Characterization Platform
}

\author{
Matthieu Picher, ${ }^{1,2}$ Stefano Mazzucco, ${ }^{1}$ Steve Blankenship, ${ }^{1}$ Glenn Holland, ${ }^{1}$ Renu Sharma ${ }^{1}$ \\ ${ }^{1}$ Center for Nanoscale Science and Technology, National Institute of Standards and Technology, \\ Gaithersburg, MD 20899-6203; \\ ${ }^{2}$ Institute for Research in Electronics and Applied Physics, University of Maryland, College park, MD \\ 20740
}

In recent years environmental transmission scanning electron microscope (ESTEM), has been successfully employed to reveal and understand the structural and chemical changes occurring in the nanoparticles under reactive environments $[1,2]$. The lack of statistical information available from TEM measurements is generally balanced by using ensemble of other techniques using $\mathrm{x}$-ray or photon sources such as diffraction, x-ray photoelectron spectroscopy, infrared spectroscopy, Raman spectroscopy etc. However, it is almost impossible to attain identical experimental set up for two separate instruments to make comparable measurements. Moreover, unambiguity of ESTEM studies may arise from the unknown effect of electron beam and the sample temperature. Here, we present a unique platform that allows us to concurrently measure atomic scale and micro-scale changes occurring in samples subjected to same reactive environmental conditions by incorporating a Raman Spectrometer on the ESTEM.

We use a parabolic mirror, attached at the end of a hollow rod that can be inserted between the sample holder and the lower pole piece of the microscope (Fig. 1-2a). The mirror focuses the incoming laser on the sample and collects the scattered Raman photons. A set of optics then carries the Raman signal up to the spectrometer. Fig. 2.b,c show the Raman D and G band as will the radial breathing modes of single walled carbon nanotubes (SWCNT) formed in the ETEM and an atomic resolution still image extracted from a video sequence, respectively. We can monitor the growth rate using G-band intensity under different growth conditions (Fig. 2d). Moreover, this versatile optical setup can also be used i) to measure the temperature using Raman shifts, ii) to investigate light/matter interactions iii) as a heating source, iii) for general spectroscopy such as cathodoluminescence. Details of design, functioning, capabilities and application to carbon nanotube synthesis will be presented.

\section{References}

[1] Sharma, R., J. Mat. Res. 2005, 20, 1695

[2] Hansen et al., Science 2001, 294, 1508 


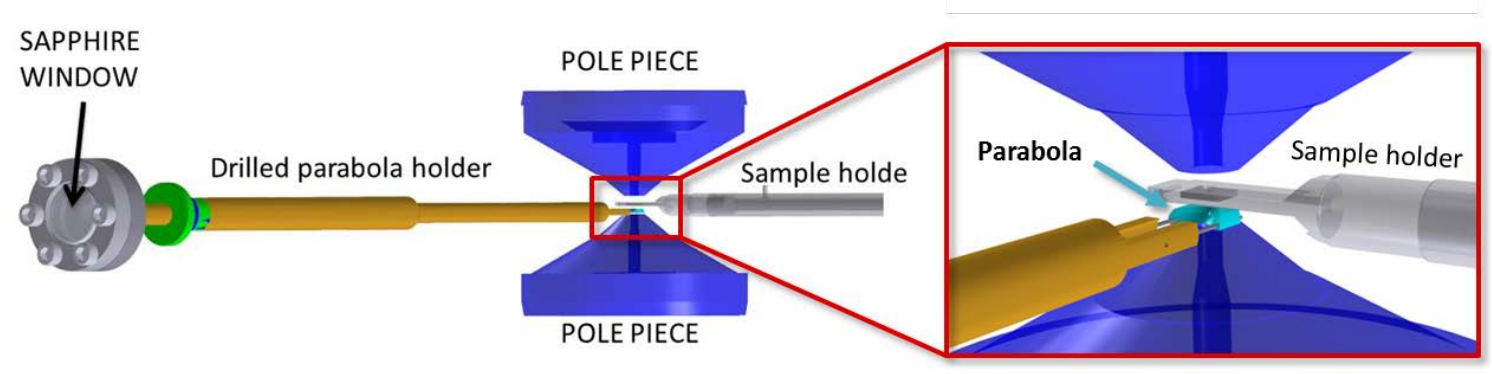

Figure 1. Schematic representation of the Raman data collection system: the laser is carried through the drilled parabola holder, then heats the parabola, is focused on the sample, and the collected Raman signal is finally sent back to the spectrometer through the parabola holder.

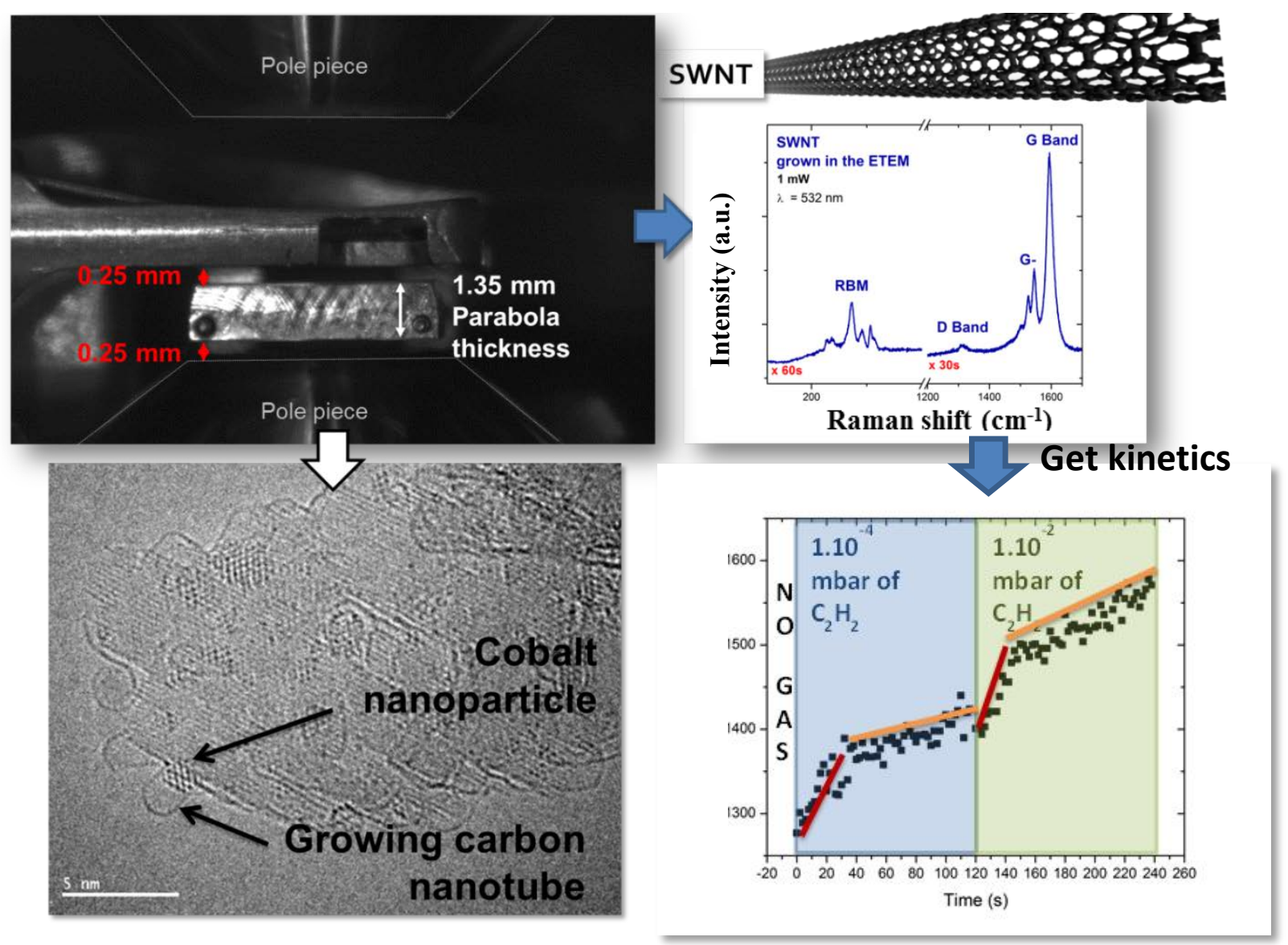

Fig. 2. (a) Location of the parabolic mirror (viewed from behind) that collects the Raman signal is inserted in between the sample holder and the lower pole piece. (b) Raman spectrum collected from SWCNTs grown in the environmental microscope. (c) Atomic Resolution ESTEM image showing growing SWCNTs attached to catalyst nanoparticle. (d) Time resolved evolution of the $\mathrm{G}$ band intensity (SWNT growth rate) at $650{ }^{\circ} \mathrm{C}$ under two different $\mathrm{C}_{2} \mathrm{H}_{2}$ pressure in the ETEM. 